\title{
SIMULATION OF RUIN PROBABILITIES FOR SUBEXPONENTIAL CLAIMS
}

\author{
By S. Asmussen and K. Binswanger
}

\begin{abstract}
We consider the classical risk model with subexponential claim size distribution. Three methods are presented to simulate the probability of ultimate ruin and we investigate their asymptotic efficiency. One, based upon a conditional Monte Carlo idea involving the order statistics, is shown to be asymptotically efficient in a certain sense. We use the simulation methods to study the accuracy of the standard Embrechts-Veraverbeke [16] approximation for the ruin probability and also suggest a new one based upon ideas of Hogan [21].
\end{abstract}

\section{KEYWORDS}

Conditional Monte Carlo, corrected diffusion approximation, ladder heights, order statistics, Pollaczeck-Khinchine formula, probability of ultimate ruin, rare events, regular variation, subexponential distribution.

\section{INTRODUCTION}

This paper is concerned with the simulation of the probability $\psi(u)$ of ruin in a classical compound Poisson risk process $U(t)$ with initial (large) reserve $u=U(0)$ in the case where the claim size distribution $B$ is heavy-tailed. Our main aim is to investigate ways to improve upon crude Monte Carlo simulation.

We assume that the claim arrival process $\{N(t), t \geq 0\} \quad(N(0)=0)$ is a homogeneous Poisson process with rate $\lambda>0$. The claim sizes are assumed to independent and identically distributed non-negative random variables $\xi_{i}(i \in N)$ with cumulative distribution function $B(x)$ and finite mean $\mu_{B}$, and independent of $\{N(t), t \geq 0\}$. The net premium is considered to be payable at a constant rate $c$ over time, where

$$
c=(1+\theta) \lambda \mu_{B}
$$

and $\theta>0$ is the relative security loading. The insurance surplus at time $t$ is $U(t)$. The total claim process $R(t)=\sum_{i=1}^{N(t)} \xi_{i}$ is by the assumptions above a compound Poisson process and thus

$$
U(t)=u+c t-R(t) .
$$

The probability of ruin is defined as

$$
\psi(u)=P\left(\inf _{t \geq 0} U(t)<0\right) .
$$

ASTIN BULLETIN, Vol. 27, No. 2, 1997, pp. 297-318 
All simulation methods that we study are based upon representing the ruin probability as $\psi(u)=z=E Z$ for some r.v. $Z$ that can be generated by simulation, simulate iid replicates $Z_{1}, \ldots, Z_{n}$ of $Z$, estimate $\psi(u)$ by $\hat{z}=\left(Z_{1}+\ldots+\right.$ $\left.Z_{n}\right) / n$ and use the empirical variance of the $Z_{i}$ to produce confidence intervals. The performance measure of a particular simulation method is the relative error $\sigma_{Z} / \psi(u)$ where $\sigma_{Z}^{2}=\operatorname{var}(Z)$ (when comparing different simulation methods based upon $Z(1), Z(2)$, say, this in only reasonable if the computer times needed to generate $Z(1), Z(2)$ are roughly the same; we assume this to be the case without further discussion). We face two difficulties:

1) The ruin problem has infinite horizon so that it is not straightforward to find the desired representation $\psi(u)=z=\mathrm{E}[Z]$ for some simulatable $Z$.

2) Since $u$ is large, the ruin probability $\psi(u)$ is small and hence we are in the framework of rare events simulation (see Heidelberger [20] or Asmussen \& Rubinstein [7] for surveys). Neglecting problem 1) for a moment, assume that we can generate $Z=I(\tau(u)<\infty)$ where $I(\cdot)$ stands for the indicator function and $\tau(u)$ is the time of ruin with initial capital $u$. This procedure is known in the literature as the the crude Monte Carlo method and leads to a relative error

$$
\frac{\sigma_{Z}}{\psi(u)}=\frac{\sqrt{\psi(u)(1-\psi(u))}}{\psi(u)} \approx \frac{1}{\sqrt{\psi(u)}} \rightarrow \infty, u \rightarrow \infty
$$

In the case where $B$ is light-tailed, a solution to both problems was suggested by Siegmund [29] and Asmussen [4] who used importance sampling (Rubinstein [28] or Glynn \& Iglehart [18]). One then performs a change of measure, replacing the given governing probability measure $P$ by a different one $\tilde{P}$ satisfying $\tilde{P}(\tau(u)<\infty)=1$ and takes $Z=d P / d \tilde{P}$ where the likelihood ratio (RadonNikodym derivative) is computed on $\mathcal{F}_{\tau(u)}$. More precisely, $\tilde{P}$ corresponds to an exponential change of measure involving the Lundberg exponent (adjustment coefficient) $R$, such that the Poisson intensity and the claim size distribution is changed in a certain way given by $R$. That problem 1) is solved follows from $\tilde{P}(\tau(u)<\infty)=1$. Empirical evidence strongly suggests that also problem 2) is solved, and the theoretical verification of this has been the subject of much research. We follow here a standard current criterion (e.g. Heidelberger [20] or Asmussen [7]) for calling a rare events simulation estimator asymptotically (or logarithmically) efficient: one should have

$$
\liminf _{u \rightarrow \infty} \frac{\log \sigma_{Z}}{\log \psi(u)} \geq 1
$$

In particular, it suffices that

$$
\sigma_{Z}^{2} \leq \psi(u)^{2} p(|\log \psi(u)|)
$$


for some polynomial $p$, and this is well-known to hold in the setting of Siegmund [29], Asmussen [4] with $p$ constant. Note that the CMC method can never be efficient according to (2) because it always gives rise to the limit $1 / 2$ rather than 1 there.

The present paper is concerned with the simulation of $\psi(u)$ in the case where $B$ does not have exponential moments so that $R$ does not exist and the method of Siegmund [29], Asmussen [4] is not applicable. Among such distributions we focus on the class of subexponential distributions $\mathcal{S}$. To be more precise:

Definition 1.1. A non negative random variable $X$ with distribution function $F$ is called subexponential $(F \in \mathcal{S})$, if for all $n \geq 2$,

$$
\lim _{x \rightarrow \infty} \frac{P\left(X_{1}+\ldots+X_{n}>x\right)}{P\left(\max \left(X_{1}, \ldots, X_{n}\right)>x\right)}=1
$$

where $X_{l}, \ldots, X_{n}$ are iid copies of $X$.

This class is quite broad and contains many of the common claim size distributions, i.e. longtailed distributions such as Pareto, Lognormal or Weibull with decreasing failure rate. Good summaries of the properties of this class are given in Embrechts and Veraverbeke [16] and Klüppelberg [23].

Our vehicle to deal with problem 1) in this setting is the Pollaczeck-Khinchine formula (see Asmussen [5])

$$
\psi(u)=1-(1-\rho) \sum_{n=0}^{\infty} \rho^{n} B_{0}^{* n}(u), u>0,
$$

where $\rho=\frac{1}{1+\ell}, B_{0}(u)=\int_{0}^{u} b_{0}(s) d s$ and $b_{0}(s)=\frac{1}{\mu_{\beta}} \bar{B}(s)$ with $\vec{B}(s)=1-B(s)$; $B_{0}^{* n}$ denotes the $n$-th convolution of $B_{0}$ with itself. Note that (4) means that $1-\psi(u)$ is a compound geometric distribution function,

$$
\psi(u)=P\left(S_{K}>u\right)
$$

where $S_{K}=X_{I}+\ldots+\ldots X_{K}, K$ is geometric with parameter $\rho$, independent of the $X_{i}$ 's, and the $X_{1}, X_{2}, \ldots$ are non-negative iid random variables with common density $b_{0}$. This means that the CMC method is applicable: $\psi(u)=z=E[Z]$ where $Z=I\left(S_{K}>u\right)$. The algorithm is as follows:

1. Generate $K_{i} \sim$ geometric $(\rho)$, i.e. $P\left(K_{i}=k\right)=(1-\rho) \rho^{k}(k=0,1,2 \ldots)$.

2. Generate $X_{1}^{i}, \ldots, X_{K_{i}}^{i}$ from the density $b_{0}$ and let $S_{K_{i}}=X_{1}^{i}+\ldots+X_{K_{i}}^{i}$.

3. If $S_{K_{i}}>u$ then $Z_{i}=1$, otherwise $Z_{i}=0$.

4. Repeat steps 1 to $3 n$ times.

5. Estimate $\mathrm{E}[Z]$ by $\hat{z}=\frac{1}{n} \sum_{i=1}^{n} Z_{i}$.

As a CMC algorithm, this procedure (referred to as Algorithm I in the following) cannot be efficient in the sense of (2). To deal with problem 2), we suggest (Section 2) two conditional Monte Carlo estimators. The idea is to replace the CMC estimator $Z$ by $E(Z \mid \mathcal{G})$ for a suitable $\sigma$-field $\mathcal{G}$, which always leads to reduction in variance, cf. Rubinstein [28]. We show that one of the estimators is efficient in the 
sense of (2) in the particular case where the tail of $B$ is regularly varying. This result is remarkable since, to our knowledge, it is the first example in the general area of rare events simulation of an asymptotically efficient solution to a problem involving heavy tails. It also has the unusual feature that the asymptotic efficient solution is not given in terms of importance sampling.

In addition to simulation methodology, we also discuss analytic approximations, of which the most standard ones are Panjer's recursion (cf. Section 4.1) and

$$
\psi(u) \sim \frac{1}{\theta} \bar{B}_{0}(u), u \rightarrow \infty
$$

(Embrechts and Veraverbeke [16] and references therein) which will be refered to as $\psi_{E V}(u)$ in the sequel. The accuracy of (6) is for instance discussed in Abate, Choudhury and Whitt [1]. They computed exact values by transform inversion (for a summary of inversion methods and applicability of this approach see Abate and Whitt [2] and references therein). In the latter paper, a class PME (Pareto Mixtures of Exponentials, see further Section 4) with explicit Laplace transforms was constructed and numerical comparisons of exact values and (6) were given with rather negative results concerning the accuracy of (6). We present some further numerical results along the same lines, computing the exact values by simulation also for more general claim size distributions than the ones in PME. Motivated by these negative findings, we suggest an alternative approximation, essentially an adaptation of the correction due to Hogan [21] of the standard diffusion approximation

$$
\psi(u) \approx \exp \left(-u \theta \frac{2 \mu_{B}}{\mu_{B}^{2}+\sigma_{B}^{2}}\right),
$$

where $\sigma_{B}^{2}$ denotes the variance of $B$. This approximation is introduced and discussed in more detail in Section 3.

\section{Conditional Monte Carlo aralgorithms}

In this section random variables are mostly denoted with capital letters (e.g. $Z, K$, $\left.S_{K}, X_{1}, X_{2}, \ldots\right)$, the realization of simulation $i(i=1, \ldots, n)$ with indexed capital letters (e.g. $Z_{i}, K_{i}, X_{1}^{i}, X_{2}^{1}, \ldots$ ).

Recall that we refer to the CMC method as Algorithm I and that a conditional Monte Carlo estimator always reduces variance.

The $95 \%$ asymptotic confidence intervals are given by:

$$
\hat{\psi}(u) \pm 1.96 \frac{\hat{\sigma}}{\sqrt{n}}
$$

where $\hat{\psi}(u)$ stands for the estimated ruin probability and $\hat{\sigma}^{2}=\frac{1}{n-1} \sum_{i=1}^{n}\left(Z_{i}-\bar{Z}\right)^{2}$. 


\subsection{Algorithm II. Write}

$$
\begin{aligned}
\psi(u) & =P\left(X_{1}+\ldots+X_{K}>u\right) \\
& =E\left[P\left(X_{1}+\ldots+X_{K}>u \mid X_{1}, \ldots, X_{K-1}\right)\right] \\
& =E\left[\bar{B}_{0}\left(u-X_{1}-\ldots-X_{K-1}\right)\right] .
\end{aligned}
$$

Thus we generate only $X_{1}, \ldots, X_{K-1}$, compute $Y=u-X_{1}-\ldots-X_{k-1}$ and set $Z=\bar{B}_{0}(Y)$, the probability that the next claim causes ruin. More precisely:

1. Generate $K_{i} \sim$ geometric $(\rho)$ i.e. $P\left(K_{i}=k\right)=(1-\rho) \rho^{k}(k=0,1,2, \ldots)$.

2. Generate $X_{1}^{i}, \ldots, X_{K_{i}}^{i}$ from the density $b_{\mathrm{o}}$ and let $Y_{i}=u-X_{1}^{i}-\ldots-X_{K_{i}-1}^{i}$.

3. Let $Z_{i}=\bar{B}_{0}\left(Y_{i}\right)\left(Z_{i}=1\right.$ if $\left.\mathrm{Y}_{i}<0\right)$

4. Repeat steps 1 to $3 n$ times.

5. Estimate $E[Z]$ by $\hat{z}=\frac{1}{n} \sum_{i=1}^{n} \mathrm{Z}_{i}$

Again $\hat{z}$ is an unbiased estimator for $\psi(u)$. However, even if the variance must be smaller than for Algorithm I, the performance as measured by (2) is not asymptotically better:

Proposition 2.1. Assume that $B \in \mathcal{S}$. Then for Algorithm II,

$$
\lim _{u \rightarrow \infty} \frac{\log \sigma_{Z}}{\log \psi(u)}=\frac{1}{2}
$$

Proof.

$$
\begin{aligned}
E\left[Z^{2}\right] & =E\left[\bar{B}_{0}^{2}\left(u-X_{1}-\ldots-X_{K-1}\right)\right] \\
& \geq E\left[\bar{B}_{0}^{2}(u) ; K \leq 1\right]+E\left[\bar{B}_{0}^{2}\left(u-X_{1}\right) ; K \geq 2\right] \\
& =\left(1-\rho^{2}\right) \bar{B}_{0}^{2}(u)+E\left[\bar{B}_{0}^{2}\left(u-X_{1}\right) ; K \geq 2\right] \\
& \geq\left(1-\rho^{2}\right) \bar{B}_{0}^{2}(u)+E\left[\bar{B}_{0}^{2}\left(u-X_{1}\right) ; X_{1}>u, K \geq 2\right]=\left(1-\rho^{2}\right) \bar{B}_{0}^{2}(u)+\rho^{2} \bar{B}_{0}(u) .
\end{aligned}
$$

The last equality follows from the fact that the event $\left(X_{I}>u\right)$ occurs with probability $\bar{B}_{0}(u)$ and then $\bar{B}_{0}^{2}\left(u-X_{1}\right)=1$. Since

$$
E[\boldsymbol{Z}]^{2}=\psi(u)^{2} \sim \frac{1}{\theta^{2}} \bar{B}_{0}^{2}(u)
$$

it follows that $\sigma_{Z}$ is of the order of magnitude at least $\bar{B}_{0}^{1 / 2} \sim(\theta \psi(u))^{1 / 2}$. Hence $\log \sigma_{Z}$ cannot go to $-\infty$ faster than $\log \psi(u) / 2$ so that $1 / 2$ is an upper bound for lim inf in (2). That $1 / 2$ is also a lower bound for lim sup follows since the 
algorithm, based upon conditional Monte Carlo, is an improvement of the CMC algorithm

2.2. Algorithm III. The third algorithm is slightly more complicated. The main idea underlying this algorithm is that for subexponential distributions only the largest claim and not the sum of all claims causes ruin as stated in Definition 1.1. The following two lemmas will elaborate on this idea.

Lemma 2.1. Let $X_{1}, X_{2}, \ldots, X_{n} \sim B_{0}$ be non negative iid random variables and denote by $X_{(1)}<X_{(2)}<\ldots<X_{(n)}$ the order statistic. Furthermore let $\mathcal{F}_{(n-1)}=$ $\sigma\left(X_{(1)}, \ldots, X_{(n-1)}\right)$.

Then

$$
P\left(X_{(n)}>x \mid \mathcal{F}_{(n-1)}\right)=\frac{\bar{B}_{0}\left(X_{(n-1)} \vee x\right)}{\bar{B}_{0}\left(X_{(n-1)}\right)}
$$

where $a \vee b$ stands for $\max (a, b)$.

Proof. Suppose $X_{1}, \ldots, X_{\mathrm{n}}$ iid and $X_{i}$ 's are absolutely continuous, then the order statistics form a Markov chain.

$$
P\left(X_{(n)}>x \mid \mathcal{F}_{(n-1)}\right)=P\left(X_{(n)}>x \mid X_{(n-1)}\right)
$$

and

$$
P\left(X_{(n)}>x \mid X_{(n-1)}=y\right)= \begin{cases}1, & x<y \\ \int_{x}^{\infty} f_{X_{(n)} \mid X_{(n-1)}}(u \mid y) d u, & x \geq y\end{cases}
$$

where

$$
\int_{x}^{\infty} f_{X_{(n)} \mid X_{n-1}}(u \mid y) d u=\frac{\bar{B}_{0}(x)}{\bar{B}_{0}(y)}
$$

(see for instance Arnold, Balakrishnan and Nagaraja [3], p. 23). Hence

$$
P\left(X_{(n)}>x \mid \mathcal{F}_{(n-1)}\right)=\frac{\bar{B}_{0}\left(X_{(n-1)} \vee x\right)}{\bar{B}_{0}\left(X_{(n-1)}\right)}
$$

Remark: If the $X_{i}$ 's are not absolutely continuous a different proof can be given using combinatorical arguments. 
Lemma 2.2. Let $S_{n}=X_{1}+\ldots+\mathrm{X}_{n}$ and $\mathrm{S}_{(k)}=X_{(1)}+\ldots+X_{(k)}(1 \leq k \leq n)$. Then

$$
P\left(S_{n}>u\right)=E\left[\frac{\bar{B}_{0}\left(\left(u-S_{(n-l)}\right) \vee X_{(n-I)}\right)}{\bar{B}_{0}\left(X_{(n-l)}\right)}\right]
$$

Proof. By conditioning,

$$
\begin{aligned}
P\left(S_{n}>u\right) & =E\left[P\left(S_{n}>u \mid \mathcal{F}_{(n-1)}\right)\right] \\
& =E\left[P\left(X_{(n)}+S_{(n-1)}>u \mid \mathcal{F}_{(n-1)}\right)\right] \\
& =E\left[P\left(X_{(n)}>u-S_{(n-1)} \mid \mathcal{F}_{(n-1)}\right)\right]
\end{aligned}
$$

and applying Lemma 2.1 completes the proof.

Algorithm III can then be written as:

1. Generate $K_{i}$ as geometric $(\rho)$, i.e. $P\left[K_{i}=k\right]=(1-\rho) \rho^{k}(k=0,1,2, \ldots)$.

2. Generate $X_{1}^{i}, \ldots, X_{K_{i}}^{i}$ from the density $b_{0}$ and set $Y^{i}=u-X_{(1)}^{i}-\ldots-X_{\left(K_{i}-1\right)}^{i}$ and $m_{i}=X_{\left(K_{i}-1\right)}^{i}$

3. Set $Z_{i}=\frac{\bar{B}_{0}\left(Y_{i} \vee m_{i}\right)}{\bar{B}_{0}\left(m_{i}\right)}$.

4. Repeat steps 1 to $3 n$ times.

5. Estimate $\mathrm{E}[\mathrm{Z}]$ by $\hat{z}=\frac{1}{n} \sum_{i=1}^{n} Z_{i}$.

The main result of the paper is the following

Theorem 2.1. Assume that $\bar{B}_{0}(x)=L(x) / x^{\alpha}(\alpha>1)$ with L slowly varying (i.e. $\lim _{x \rightarrow x} \frac{L(\lambda x)}{L(x)}=1$ for all $\left.\lambda>0\right)$. Then Algorithm III satisfies

$$
\liminf _{u \rightarrow \infty} \frac{\log \sigma_{Z}}{\log \psi(u)} \geq 1
$$

In order to proof Theorem 2.1 we first give three Lemmas.

Lemma 2.3. For Algorithm III we have

$$
\sigma_{Z}^{2} \leq E\left[K^{2}\left(\frac{1}{2} \bar{B}_{0}^{2}\left(\frac{u}{2}\right)+\bar{B}_{0}^{2}\left(\frac{u}{K}\right)\left|\log \bar{B}_{0}\left(\frac{u}{2}\right)\right|\right)\right]
$$

Proof. We first derive the conditional density $f_{X_{(K-i)}}(x)$ of the random variable $\left.X_{(K} 1\right)$ given $K$ :

$$
\begin{aligned}
P\left(X_{K-1} \leq x\right) & =P\left(X_{(1)} \leq x, \ldots, X_{(K-1)} \leq x\right) \\
& =\left(\begin{array}{c}
K \\
1
\end{array}\right) P\left(X_{1} \leq x, \ldots, X_{K-1} \leq x, X_{K}>x\right)
\end{aligned}
$$




$$
\begin{aligned}
& +P\left(X_{1} \leq x, \ldots, X_{K-1} \leq x, X_{K} \leq x\right) \\
= & K B_{0}^{K-1}(x) \bar{B}_{0}(x)+B_{0}^{K}(x) .
\end{aligned}
$$

Hence the density is:

$$
f_{X_{(K-1)}}(x)=K(K-1) B_{0}^{K-2}(x) \bar{B}_{0}(x) b_{0}(x) .
$$

Next we calculate

$$
\begin{aligned}
E\left[Z^{2} \mid K\right]= & E\left[\left(\frac{\bar{B}_{0}\left(\left(u-S_{(K-1)}\right) \vee X_{(K-1)}\right)}{\bar{B}_{0}\left(X_{(K-1)}\right)}\right)^{2} \mid K\right] \\
= & E\left[\left(\frac{\bar{B}_{0}\left(u-S_{(K-1)}\right)}{\bar{B}_{0}\left(X_{(K-1)}\right)}\right)^{2} ; X_{(K-1)} \leq \frac{u}{K} \mid K\right] \\
& +E\left[\left(\frac{\bar{B}_{0}\left(\left(u-S_{(K-1)}\right) \vee X_{(K-1)}\right)}{\bar{B}_{0}\left(X_{(K-1)}\right)}\right)^{2} ; \frac{\mathrm{u}}{K}<X_{(K-1)} \leq \frac{u}{2} \mid K\right] \\
& +E\left[1 ; X_{(K-1)}>\frac{u}{2} \mid K\right] .
\end{aligned}
$$

The first summand (10) can be bounded as follows. If $X_{(K-1)} \leq \frac{u}{K}$ then $\bar{B}_{0}\left(u-S_{(K-1)}\right) \leq \bar{B}_{0}\left(\frac{u}{K}\right)$, so that

$$
\begin{gathered}
E\left[\left(\frac{\bar{B}_{0}\left(u-S_{(K-1)}\right)}{\bar{B}_{0}\left(X_{(K-1)}\right)}\right)^{2} ; X_{(K-1)} \leq \frac{u}{K} \mid K\right] \\
\leq \bar{B}_{0}^{2}\left(\frac{u}{K}\right) \int_{0}^{u / K} \frac{f_{X_{(K-1)}}(x)}{\bar{B}_{0}^{2}(x)} d x \\
\leq K(K-1) \bar{B}_{0}^{2}\left(\frac{u}{K}\right) \int_{0}^{u / K} \frac{b_{0}(x)}{\bar{B}_{0}(x)} d x \\
=-K(K-1) \bar{B}_{0}^{2}\left(\frac{u}{K}\right) \log \left(\bar{B}_{0}\left(\frac{u}{K}\right)\right) .
\end{gathered}
$$


The second summand (11) can be bounded in the same way. For $\frac{u}{K}<X_{(K-1)} \leq \frac{u}{2}, \bar{B}_{0}\left(\left(u-S_{(K-1)}\right) \vee X_{(K-1)}\right) \leq \bar{B}_{0}\left(\frac{u}{K}\right)$, yielding

$$
\begin{gathered}
E\left[\left(\frac{\bar{B}_{0}\left(\left(u-S_{(K-1)}\right) \vee X_{(K-1)}\right)}{\bar{B}_{0}\left(X_{(K-1)}\right)}\right)^{2} ; \frac{u}{K}<X_{(K-1)} \leq \frac{u}{2} \mid K\right] \\
\leq \bar{B}_{0}^{2}\left(\frac{u}{K}\right) \int_{u / K}^{u / 2} \frac{f_{X_{(K-1)}}(x)}{\bar{B}_{0}^{2}(x)} d x \\
\leq K(K-1) \bar{B}_{0}^{2}\left(\frac{u}{K}\right) \int_{u / K}^{u / 2} \frac{b_{0}(x)}{\bar{B}_{0}(x)} d x \\
=-K(K-1) \bar{B}_{0}^{2}\left(\frac{u}{K}\right)\left(\log \left(\bar{B}_{0}\left(\frac{u}{2}\right)\right)-\log \left(\bar{B}_{0}\left(\frac{u}{K}\right)\right)\right)
\end{gathered}
$$

To find an upper bound for (12) we write

$$
\begin{aligned}
E\left[1 ; X_{(K-1)}>\frac{u}{2} \mid K\right] & =\int_{u / 2}^{\infty} f_{X_{(K-1)}}(x) d x \\
& =K(K-1) \int_{u / 2}^{\infty} B_{0}^{K-2}(x) \bar{B}_{0}(x) b_{0}(x) d x \\
& \leq K(K-1) \int_{u / 2}^{\infty} \bar{B}_{0}(x) b_{0}(x) d x \\
& =K(K-1) \frac{1}{2} \bar{B}_{0}^{2}\left(\frac{u}{2}\right) .
\end{aligned}
$$

Adding the above inequalities leads to

$$
\begin{aligned}
E\left[Z^{2} \mid K\right] & \leq K(K-1)\left(\frac{1}{2} \bar{B}_{0}^{2}\left(\frac{u}{2}\right)-\bar{B}_{0}^{2}\left(\frac{u}{K}\right) \log \left(\bar{B}_{0}\left(\frac{u}{2}\right)\right)\right) \\
& \leq K^{2}\left(\frac{1}{2} \bar{B}_{0}^{2}\left(\frac{u}{2}\right)+\bar{B}_{0}^{2}\left(\frac{u}{K}\right)\left|\log \bar{B}_{0}\left(\frac{u}{2}\right)\right|\right)
\end{aligned}
$$

and hence

$$
\begin{aligned}
\sigma_{Z}^{2} & =E\left[E\left[Z^{2} \mid K\right]\right]-E[Z]^{2} \\
& \leq E\left[K^{2}\left(\frac{1}{2} \bar{B}_{0}^{2}\left(\frac{u}{2}\right)+\bar{B}_{0}^{2}\left(\frac{u}{K}\right)\left|\log \bar{B}_{0}\left(\frac{u}{2}\right)\right|\right)\right] .
\end{aligned}
$$


Lemma 2.4. If $\bar{B}_{0}(x)=\frac{L(x)}{x^{a}}, L$ slowly varying then for any $\varepsilon>0$ there exist constants $C_{-}(\varepsilon)$ and $C_{+}(\varepsilon)$ such that

$$
C_{-}(\varepsilon) d^{\alpha-\varepsilon} x^{-\alpha-\varepsilon} \leq \bar{B}_{0}\left(\frac{x}{d}\right) \leq C_{+}(\varepsilon) d^{\alpha-\varepsilon} x^{-\alpha-\varepsilon}, \forall x>0 \forall d>0 .
$$

Proof. From $\bar{B}_{0}(x) x^{\alpha-\varepsilon}=x^{-\varepsilon} L(x)$ it follows that $\lim _{x \rightarrow 0} x^{-\varepsilon} L(x)=0$ and that $L$ is a continuous function. Since $L$ is slowly varying also $\lim _{x \rightarrow \infty} x^{-\varepsilon} L(x)=0$. Hence there exists a constant $C_{+}(\varepsilon)$ such that $L(x) \leq C_{+}(\varepsilon) x^{\varepsilon}$ for all $x$ and hence

$$
\bar{B}_{0}\left(\frac{x}{d}\right)=\frac{L(x / d)}{(x / d)^{\alpha}} \leq \frac{C_{+}(\varepsilon)(x / d)^{\varepsilon}}{(x / d)^{\alpha}}
$$

For the lower bound the proof is similar. Just note that if $L$ is slowly varying then also $1 / \mathrm{L}$ is slowly varying.

Lemma 2.5. If $\bar{B}_{0}(x)=\frac{L(x)}{x^{*}}, L$ slowly varying then for any $\varepsilon>0$ there exist constants $D_{1}(\varepsilon)$ and $D_{2}(\varepsilon)$ such that

$$
E\left[Z^{2}\right] \leq\left(D_{1}(\varepsilon)+D_{2}(\varepsilon)|\log u|\right) u^{2 \varepsilon-2 \alpha x} .
$$

Proof. From Lemma 2.3 we have

$$
E\left[Z^{2}\right] \leq E\left[K^{2}\left(\frac{1}{2} \bar{B}_{0}^{2}\left(\frac{u}{2}\right)+\bar{B}_{0}^{2}\left(\frac{u}{K}\right)\left|\log \bar{B}_{0}\left(\frac{u}{2}\right)\right|\right)\right]
$$

Lemma 2.4 yields

$$
\begin{aligned}
\leq & E\left[K^{2}\right] \frac{1}{2} C_{+}^{2}(\varepsilon) 2^{2 \alpha-2 \varepsilon} u^{-2 \alpha+2 \varepsilon} \\
& +E\left[C_{+}^{2}(\varepsilon) K^{2 \alpha-2 \varepsilon+2} u^{-2 \alpha+2 \varepsilon}\left|\log \left(C_{-}(\varepsilon) 2^{\alpha-\varepsilon} u^{-\alpha-\varepsilon}\right)\right|\right] \\
\leq & \left(D_{1}(\varepsilon)+D_{2}(\varepsilon)|\log u|\right) u^{2 \varepsilon-2 \alpha}
\end{aligned}
$$

where $D_{1}(\varepsilon)=E\left[K^{2}\right] \frac{1}{2} C_{+}^{2}(\varepsilon) 2^{2 \alpha-2 \varepsilon}+E\left[K^{2 \alpha-2 \varepsilon+2}\right] C_{+}^{2}(\varepsilon)\left|\log \left(C_{-}(\varepsilon) 2^{\gamma-\varepsilon}\right)\right|$

and $D_{2}(\varepsilon)=E\left[K^{2 \alpha-2 \varepsilon+2}\right] C_{+}^{2}(\varepsilon)(\alpha+\varepsilon)$.

Now we have all the tools needed to prove Theorem 2.1 .

Proof of Theorem 2.1. From Lemma 2.5 we get

$$
\begin{aligned}
\log \sigma_{Z} & \leq \log \sqrt{\left(D_{1}(\varepsilon)+D_{2}(\varepsilon)|\log u|\right) u^{2 \varepsilon-2 \alpha}} \\
& =\frac{1}{2} \log \left(D_{1}(\varepsilon)+D_{2}(\varepsilon)|\log u|\right)+(\varepsilon-\alpha) \log u
\end{aligned}
$$


and therefore

$$
\lim _{u \rightarrow \infty} \frac{\log \sigma_{Z}}{\log \psi(u)} \geq \lim _{u \rightarrow \infty} \frac{\frac{1}{2} \log \left(D_{1}(\varepsilon)+D_{2}(\varepsilon)|\log u|\right)+(\varepsilon-\alpha) \log u}{\log \psi(u)}
$$

using (6) yields

$$
\begin{aligned}
& =\lim _{u \rightarrow \infty} \frac{\frac{1}{2} \log \left(\mathrm{D}_{1}(\varepsilon)+\mathrm{D}_{2}(\varepsilon)|\log \mathrm{u}|\right)+(\varepsilon-\alpha) \log \mathrm{u}}{\log \left(\bar{B}_{0}(u) / \theta\right)} \\
& =\lim _{u \rightarrow \infty} \frac{\frac{1}{2} \log \left(\mathrm{D}_{1}(\varepsilon)+\mathrm{D}_{2}(\varepsilon)|\log \mathrm{u}|\right)+(\varepsilon-\alpha) \log \mathrm{u}}{-\log \theta+\log \mathrm{L}(\mathrm{u})-\alpha \log \mathrm{u}} \\
& =\frac{\varepsilon-\alpha}{-\alpha}=1-\frac{\varepsilon}{\alpha}
\end{aligned}
$$

Now let $\varepsilon \rightarrow 0$ which completes the proof.

\section{Remark:}

1. For lognormal claimsizes Algorithm III is also asymptotically efficient. The proof is given in Binswanger [8].

\begin{tabular}{|c|c|c|c|c|}
\hline \multicolumn{5}{|c|}{ Pareto $(1,2), \theta=0.1, n=1000$} \\
\hline $\begin{array}{l}\hat{\psi}(u) \pm 1.96 \frac{\hat{\sigma}}{\sqrt{n}} \\
\log (\hat{\sigma}) / \log (\hat{\psi})\end{array}$ & Algorithm I & Algorithm II & Algorithm III & $\psi_{P}(u)$ \\
\hline $\mathrm{u}=10$ & $(5.6 \pm \underset{1.21}{0.3) \cdot 10}$ & $\frac{\left(6.0 \pm \underset{1.56}{0.3)} \cdot 10^{-1}\right.}{}$ & $\underset{1.38}{(5.5 \pm 0.3) \cdot 10^{-1}}$ & $5.5 \cdot 10^{1}$ \\
\hline $\mathrm{u}=50$ & $\begin{array}{c}(2.0 \pm 0.2) \cdot 10^{-1} \\
0.57\end{array}$ & $\begin{array}{c}(2.0 \pm 0.2) \cdot 10^{-1} \\
0.60\end{array}$ & $\begin{array}{c}(1.9 \pm 0.2) \\
0.72\end{array}$ & $1.9 \cdot 10^{-1}$ \\
\hline $\mathrm{u}=100$ & $\underset{0.52}{(8.1 \pm 1.7) \cdot 10^{2}}$ & $\underset{0.54}{(9.0 \pm 1.7) \cdot 10^{2}}$ & $\underset{0.69}{(8.6 \pm 1.2) \cdot 10^{-.2}}$ & $8.5 \cdot 10^{-2}$ \\
\hline $\mathrm{u}=500$ & $\begin{array}{c}(1.2 \pm 0.7) \cdot 10^{2} \\
0.50\end{array}$ & $\begin{array}{c}(0.9 \pm 0.5) \cdot 10^{2} \\
0.51\end{array}$ & $\begin{array}{c}(1.0 \pm 0.2) \cdot 10^{2} \\
0.77\end{array}$ & $1.2 \cdot 10^{-2}$ \\
\hline$u=1000$ & $\begin{array}{c}(6.0 \pm 4.8) \cdot 10^{3} \\
0.50\end{array}$ & $\begin{array}{c}(9.5 \pm 5.9) \cdot 10^{-3} \\
0.51\end{array}$ & $\begin{array}{c}(5.3 \pm 0.6) \cdot 10^{3} \\
0.88\end{array}$ & $5.4 \cdot 10^{3}$ \\
\hline
\end{tabular}

TABLE 1

SIMULATHD RUIN PROBABILITIES AND THEIR PRECISION MEASURED BY (2) FOR PARETO DISTRIBUTED ( LAIMS (ALL NUMBERS ARE ROUNDED TO THEIR LAST DIGIT). 
TABLE 2

SIMULATED RUIN PROBABILITIES AND THEJR PRECISION MEASURED BY (2) FOR PME DISTRIBUTED CLAIMS (ALL NUMBERS ARE ROUNDED TO THEIR LAST DIGIT)

\begin{tabular}{|c|c|c|c|c|}
\hline \multicolumn{5}{|c|}{$\operatorname{PME}(3), \theta=0.25, n=1000$} \\
\hline $\begin{array}{l}\hat{\psi}(u) \pm 1.96 \frac{\hat{\sigma}}{\sqrt{n}} \\
\log (\hat{\sigma}) / \log (\hat{\psi})\end{array}$ & Algorithm I & Algorithm II & Algorithm III & $\psi(u)$ \\
\hline $\mathrm{u}=50$ & $\begin{array}{c}(5.0 \pm 4.4) \cdot 10^{-3} \\
0.50\end{array}$ & $\underset{0.54}{\left(1.8 \pm \underset{0}{2.0)} \cdot 10^{3}\right.}$ & $\begin{array}{c}(3.0=0.9) \cdot 10^{3} \\
0.74\end{array}$ & $3.1 \cdot 10^{3}$ \\
\hline$u=60$ & $\begin{array}{c}(3.0 \pm 3.4) \cdot 10^{3} \\
0.50\end{array}$ & $\begin{array}{c}(4.3 \pm 3.9) \cdot 10^{3} \\
0.51\end{array}$ & $\left(2.4 \pm \underset{0.57}{2.0)} \cdot 10^{3}\right.$ & $1.8 \cdot 10^{3}$ \\
\hline $\mathrm{u}=70$ & $\begin{array}{c}\left(2.0 \pm \underset{0.50}{2.8)} \cdot 10^{3}\right. \\
.3\end{array}$ & $\begin{array}{c}(1.8 \pm 0.1) \cdot 10^{3} \\
1.04\end{array}$ & $\begin{array}{c}(1.0 \pm 0.2) \cdot 10^{3} \\
0.84\end{array}$ & $1.2 \cdot 10^{3}$ \\
\hline $\mathrm{u}=80$ & 0 & $\begin{array}{c}\left(1.4 \pm \underset{1.05}{0.1)} \cdot 10^{4}\right. \\
1.05\end{array}$ & $\underset{0.82}{(8.8 \pm 2.0) \cdot 10^{4}}$ & $8.2 \cdot 10^{4}$ \\
\hline $\mathrm{u}=90$ & $\begin{array}{c}(1.0 \pm 2.0) \cdot 10^{-3} \\
0.50\end{array}$ & $\begin{array}{c}(1.0 \pm 0.1) \cdot 10^{4} \\
1.01\end{array}$ & $\underset{0.84}{(5.6 \pm 1.1) \cdot 10^{4}}$ & $6.1 \cdot 10^{4}$ \\
\hline $\mathrm{u}=100$ & $\underset{0.50}{(1.0 \pm 2.0) \cdot 10^{-3}}$ & $\left(8.2 \pm \underset{1.06}{0.3)} \cdot 10^{5}\right.$ & $\begin{array}{c}(4.1 \pm 0.7) \cdot 10^{4} \\
0.87\end{array}$ & $4.7 \cdot 10^{4}$ \\
\hline
\end{tabular}

TABLE 3

SIMULATED RUIN PROBABILITIES AND THEIR PRECISION MEASURED BY (2) IOR LOGNORMAL DISTRIBUTED CLAIMS (ALL NUMBERS ARE ROUNDED TO THEIR LAST DIGIT)

\begin{tabular}{|l|c|c|c|c|}
\hline \multicolumn{5}{|c|}{ Lognormal (-1.62, 1.8), $\theta=0.1, n=1000$} \\
\hline $\begin{array}{l}\hat{\psi}(u) \pm 1.96 \frac{\hat{\sigma}}{\sqrt{n}} \\
\log (\hat{\sigma}) / \log (\hat{\psi})\end{array}$ & Algorithm I & Algorithm II & Algorithm III & $\psi(u)$ \\
\hline $\mathrm{u}=0$ & $\begin{array}{c}(8.3 \pm 0.2) \cdot 10^{-1} \\
5.35\end{array}$ & $\begin{array}{c}(8.9 \pm 0.2) \cdot 10^{1} \\
11.1\end{array}$ & $\begin{array}{c}(9.0 \pm 0.2) \cdot 10^{1} \\
11.4\end{array}$ & $9.1 \cdot 10^{1}$ \\
\hline $\mathrm{u}=100$ & $\begin{array}{c}(3.5 \pm 0.3) \cdot 10^{-1} \\
0.70\end{array}$ & $\begin{array}{c}(3.9 \pm 0.3) \cdot 10^{1} \\
0.82\end{array}$ & $\begin{array}{c}(3.4 \pm 0.3) 10^{1} \\
0.84\end{array}$ & $3.4 \cdot 10^{1}$ \\
\hline $\mathrm{u}=1000$ & $\begin{array}{c}(1.2 \pm 0.7) \cdot 10^{2} \\
0.50\end{array}$ & $\begin{array}{c}(7.4 \pm 4.8) \cdot 10^{3} \\
0.52\end{array}$ & $\begin{array}{c}(8.0 \pm 2.2) \cdot 10^{3} \\
0.69\end{array}$ & $1.1 \cdot 10^{2}$ \\
\hline $\mathrm{u}=10000$ & 0 & $(3.3 \pm 0.1) \cdot 10^{-6}$ & $(3.5 \pm 0.4) \cdot 10^{5}$ & $4 \cdot 10^{5}$ \\
\hline
\end{tabular}


2. If $B_{0}($ or $B)$ is a Weibull distribution,

$$
b_{0}(x)=v x^{\nu-1} e^{-x^{r}}, \bar{B}_{0}(x)=e^{-x^{\prime}},
$$

Algorithm III is not efficient in the sense of (2). Indeed, we get

$$
\begin{aligned}
E\left[Z^{2} \mid K=2\right] & \geq \int_{0}^{u / 2} \frac{\bar{B}_{0}^{2}(u-y)}{\bar{B}_{0}^{2}(y)} f_{X_{(1)}}(y) d y \\
& =2 \int_{0}^{u / 2} \bar{B}_{0}^{2}(u-y) v y^{v-1} d y \\
& \geq 2 v(u / 2)^{v-1} \int_{0}^{u / 2} \bar{B}_{0}^{2}(u-y) d y \\
& =2 v(u / 2)^{v-1} \int_{u / 2}^{u} \bar{B}_{0}^{2}(y) d y \\
& \geq \int_{u / 2}^{u} 2 v y^{v-1} \bar{B}_{0}^{2}(y) d y \\
& =e^{-2(u / 2)^{v}-e^{-2 u^{v}}=e^{-2^{1-u^{\prime}} u^{r}}(1+o(1))}
\end{aligned}
$$

So we get

$$
\begin{aligned}
\lim _{u \rightarrow \infty} \frac{\log \sigma_{Z}}{\log \psi(u)} & \leq \lim _{u \rightarrow \infty} \frac{\frac{1}{2} \log \left(E\left[Z^{2} \mid K=2\right] P(K=2)-E\left[Z^{2}\right]\right)}{\log \left(\bar{B}_{0}(u) / \theta\right)} \\
& \leq \lim _{u \rightarrow \infty} \frac{2^{1-v} u^{v} / 2}{u^{v}}=\frac{1}{2^{v}}<1
\end{aligned}
$$

Of course we should mention that this does not imply that the algorithm does not work well in the Weibull setting; and indeed the numerical experience is convincing. It should be noted that, as a conditional $\mathrm{MC}$ algorithm, Algorithm III is always an improvement on the crude MC method, even in the light tailed case. (Though here we do not obtain any improvement of the asymptotic efficiency and the algorithms of Asmussen [4], Siegmund [29] are superior.)

\section{THE CORRECTED DIFFUSION APPROXIMATION}

The standard diffusion approximation (Iglehart [22] or Grandell [19]) is given by (7). For light-tailed random walk problems Siegmund [30] derived a correction which was adapted to ruin probabilities by Asmussen [6] and shown to be extremely accurate. An alternative covering also certain heavy-tailed cases was given in Theorem 2 of Hogan [21]. As in Asmussen [6], it requires some 
adaptation to ruin probabilities which we shall next present. The result will be an approximation of the type

$$
\psi_{H}(u)=\exp \left(-c_{1} u\right)\left(1+c_{2} u-c_{3}\right)
$$

where

$$
c_{1}=\frac{2 \theta m_{1}}{m_{2}}, c_{2}=\frac{4 \theta^{2} m_{1}^{2} m_{3}}{3 m_{2}^{3}}, c_{3}=\frac{2 \theta m_{1} m_{3}}{3 m_{2}^{2}}
$$

and $m_{i}$ is the $i$-th moment of $B$. Note that formally the conditions of Theorem 2 in Hogan [21] lead to the requirement that $m_{5}<\infty$ though our numerical experience indicates that this is not crucial.

To derive (13) from Hogan [21], substitute first $v=\xi / \vartheta$ to get

$$
P_{-\vartheta}\left(\tau_{v}<\infty\right) \approx e^{-2 \vartheta v}\left(1+\frac{4 \gamma \vartheta^{2} v}{3}-\frac{\vartheta E_{0} S_{\tau+}^{2}}{E_{0} S_{\tau+}}\right) .
$$

Next we consider a RW with drift $-\mu$ and $\sigma^{2}=E_{0} X_{1}^{2}$ not necessarily $=1$, and $\tau_{u}$. The normalized RW $S_{n} / \sigma$ has drift $-\vartheta=-\mu / \sigma, \gamma=E_{0} X_{1} / \sigma^{3}, v=u / \sigma$. Similar substitutions for the ladder height moments yield

$$
\begin{aligned}
P_{-\mu}\left(\tau_{u}<\infty\right) & \approx e^{-2 \mu u / \sigma^{2}}\left(1+\frac{4 E_{0} X_{1}^{3} / \sigma^{3} \cdot \mu^{2} / \sigma^{2} \cdot u / \sigma}{3}-\frac{\mu / \sigma \cdot E_{0} S_{\tau+1}^{2} / \sigma^{2}}{E_{0} S_{\tau+} / \sigma}\right) \\
& =e^{-\left(2 \mu / \sigma^{2}\right) u}\left(1+\frac{4 E_{0} X_{1}^{3} \mu^{2}}{3 \sigma^{6}} u-\frac{\mu E_{0} S_{\tau \cdot+}^{2}}{\sigma^{2} E_{0} S_{\tau+}}\right) .
\end{aligned}
$$

In the next step, we take the RW as a discrete skeleton of the risk process, $S_{n}=R(n h)-c n h$. Then

$$
\mu=h \theta \lambda m_{1}, \quad \sigma^{2}=h \lambda m_{2}, \quad E_{0} X_{1}^{3}=h \lambda m_{3} .
$$

Further the risk process corresponding to $\vartheta=0$ has ladder height distribution $B_{\theta}$ so that

$$
\frac{E_{0} S_{\tau+}^{2}}{E_{0} S_{\tau+}} \rightarrow \frac{\int_{0}^{\infty} x^{2} B_{0}(d x)}{\int_{0}^{\infty} x B_{0}(d x)}=\frac{2 m_{3}}{3 m_{2}}, h \downarrow 0
$$

Taking the limit $h \downarrow 0$ in (15) we thus get

$$
\begin{aligned}
\psi(u) & \approx e^{-\left(2 \theta \lambda m_{1} / \lambda m_{2}\right) u}\left(1+\frac{4 \lambda m_{3}\left(\theta \lambda m_{1}\right)^{2}}{3 \lambda^{3} m_{2}^{3}} u-\frac{2 m_{3} \theta \lambda m_{1}}{3 \lambda m_{2}^{2}}\right) \\
& =e^{-c_{1} u}\left(1+c_{2} u-c_{3}\right) .
\end{aligned}
$$


Various other approximations and bounds for $\psi(u)$ are known. For an overview see Embrechts and Klüppelberg [15], Feilmeier and Bertram [17], Panjer [26], Buchwald, Chevallier and Klüppelberg [9] and references therein.

\section{NUMERICAL RESULTS}

In this section we present the numerical evaluation of the algorithms for the PME, the Pareto and the Lognormal case. For the PME distributions Abate, Choudhury and Whitt [1] have calculated the exact values of the ruin probabilities. Therefore we choose the parameters in such a way that we can compare the simulation and the exact results. For the Pareto and the Lognormal case only few exact values are available. The Panjer approximation $\psi_{P}(u)$ (see below) is chosen as a benchmark.

The simulation has been done with MATLAB 4.2a. To construct $B_{0}$ distributed random variables we used the inversion method for the Pareto case and the inversion/rejection method by Newton-Raphson iteration for the other two. For more details see for instance Devroye [11].

4.1. The Panjer recursion. Panjer [27] suggested to use a recursion formula for calculating the probability of ultimate survival $\phi=1-\psi$. The recursion formula is based on a discretisation of the density $\phi^{\prime}$ which we denote by $\phi^{*}$ leading to

$$
\phi^{*}(u)=\frac{1}{1+\theta-g(0)} \sum_{y=1}^{u} g(y) \phi^{*}(u-y) \quad u=1,2, \ldots
$$

with

$$
\phi^{*}(0)=\frac{\theta}{1+\theta-g(0)}
$$

where $g$ is a discretised version of the density $b_{0}$. Finally we get

$$
\psi(u) \approx 1-\sum_{y=0}^{u} \phi^{*}(y) \quad u=0,1,2, \ldots
$$

The time to evaluate this procedure increases for large $u$ since the recursion always has to start with $u=0$. A great advantage of this method is that it leads to upper and lower bounds for $\psi(u)$ by choosing $g$ in such a way that $g_{l}(x) \leq b_{0}(x)$ for the lower bound and $g_{u}(x) \geq b_{0}(x)$ for the upper bound. Since $b_{0}$ is a decreasing function we can set $g_{l}(x)=B_{0}([x]+1)-B_{0}([x])$ and $g_{u}(x)=$ $B_{0}([x])-B_{0}([x]-1)([x]$ stands for the integer part of $x)$. For the approximation of $\psi(u)$ denoted by $\psi_{P}(u)$ we choose $g_{a}(x)=B_{0}([x]+1 / 2)-B_{0}([x]-1 / 2)$. Panjer's recursion method has meanwhile become the standard tool for actuaries; see for instance Dickson [13] for a comprehensive review. 
4.2. Pareto Distribution (PAR $(a, b))$ ). The distribution function of the Pareto distribution is given by:

$$
B(x)=\left(1-\left(\frac{a}{x}\right)^{b}\right) I(x>a) \text { where } a>0, b>1, \text { and } x>0 .
$$

The mean is $\mu_{B}=a b /(b-1)$, and the density $b_{0}$ and the cdf $B_{0}$ of the integrated tail distribution are respectively

$$
\begin{gathered}
b_{0}(x)=\frac{b-1}{a b}\left(I(x<a)+\left(\frac{a}{x}\right)^{b} I(x \geq a)\right), \\
B_{0}(x)=\frac{b-1}{a b} x I(x<a)+\left(1-\frac{1}{b}\left(\frac{a}{x}\right)^{b-1}\right) I(x \geq a) .
\end{gathered}
$$

For the simulation with the inversion method we also need $B_{0}^{-1}(x)$ which is

$$
B_{0}^{-1}(x)=\frac{a b}{b-1} x I\left(x<\frac{b-1}{b}\right)+\frac{a}{(b(1-x))^{\frac{1}{h-1}}} I\left(x \geq \frac{b-1}{b}\right) .
$$

4.3. Pareto Mixture of Exponentials Distribution (PME( $r)$ ). This class of distribution was defined in order to have subexponential distributions with an explicit Laplace transform. Starting from a Pareto distribution the PME is defined as follows.

Definition 4.1. Let for $r>1$

$$
f(x)=r\left(\frac{r-1}{r}\right)^{r} x^{-(r+1)} I\left(x>\frac{r-1}{r}\right)
$$

be the density function of a Pareto distribution with mean 1. Then the density of a Pareto Mixture of Exponentials is defined as

$$
b(x):=\int_{\frac{r-1}{r}}^{\infty} f(y) \frac{1}{y} \exp \left(-\frac{x}{y}\right) d y=\left(\frac{r-1}{r}\right)^{r} x^{-(r+1)} \gamma\left(r+1, \frac{r}{r-1} x\right),
$$

where $\gamma(a, u)=\int_{o}^{u} t^{a-1} \exp (-t) d t$ is the incomplete Gamma function.

The tail behavior of the density of a PME distribution is the same as for the Pareto distribution, namely $\sim c_{r} x^{-(r+1)}$ ( $c_{r}$ a constant depending only on $r$ ). The distribution function $B_{0}(x)$ can be calculated explicitly for some values of $r$, for example for $r=3$ :

$$
B_{0}(x)=1-\frac{1}{9 x^{2}}\left(8-(8+12 x) \exp \left(-\frac{3 x}{2}\right)\right)
$$


4.4. Lognormal Distribution ( $\mathbf{L N}(m, s))$. The density of a Lognormal distribution is given by

$$
b(x)=\frac{1}{s \sqrt{2 \pi}} \frac{1}{x} \exp \left(-\frac{1}{2}\left(\frac{\log (x)-m}{s}\right)^{2}\right) \quad I(x>0)
$$

and the k-th moment $\mu_{B}^{(k)}=\exp \left(k m+\frac{1}{2} k^{2} s^{2}\right) . \quad B(x)=\Phi(w(x))$ where $\Phi(\cdot)$ denotes the c.d.f. of a standard normal distribution and $w(x)=\frac{1}{s}(\log (x)-m)$. For efficient programming the following representation of $B_{0}(x)$ is useful:

$$
\begin{aligned}
B_{0}(u) & =\frac{1}{\mu_{B}} \int_{0}^{u}(1-B(x)) d x \\
& =\frac{1}{\mu_{B}}\left(u-\frac{1}{\sqrt{2 \pi}} \int_{0}^{u} \int_{-\infty}^{w(u)} \exp \left(-y^{2} / 2\right) d y d x\right) \\
& =\frac{1}{\mu_{B}}\left(u-\frac{1}{\sqrt{2 \pi}} \int_{-\infty}^{w(u)} \int_{w^{-1}(y)}^{u} \exp \left(-y^{2} / 2\right) d x d y\right) \\
& =\frac{1}{\mu_{B}}\left(u-u \Phi(w(u))+\mu_{B} \Phi(w(u)-s)\right) .
\end{aligned}
$$

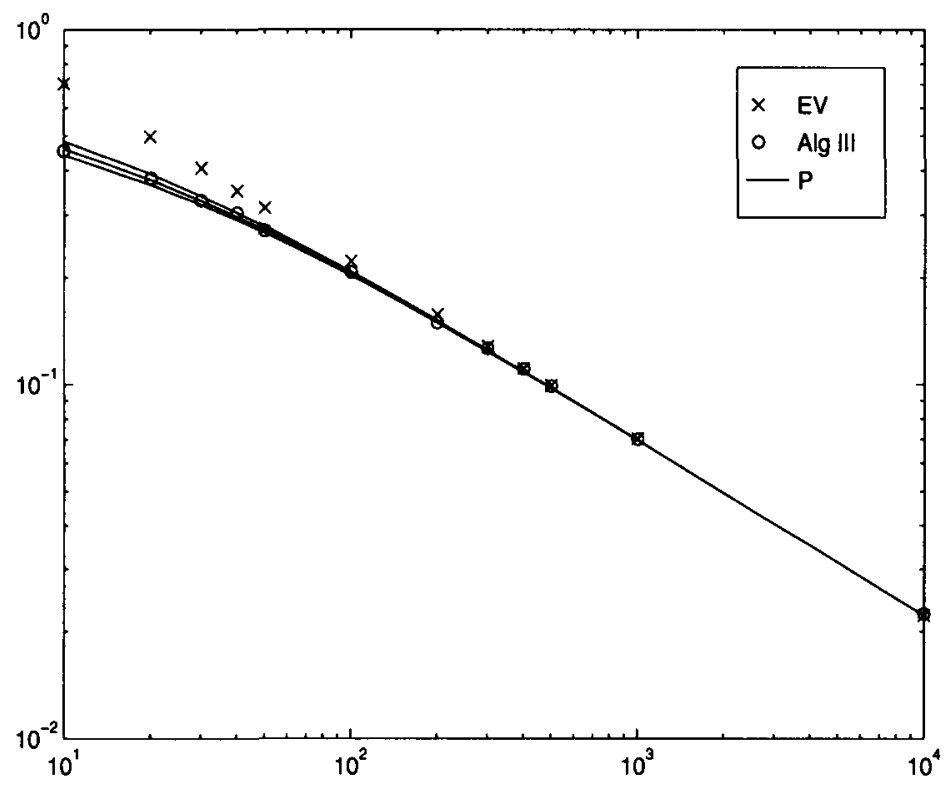

FIGURE 1: PAR(1,1.5), $\theta=0.3$. 


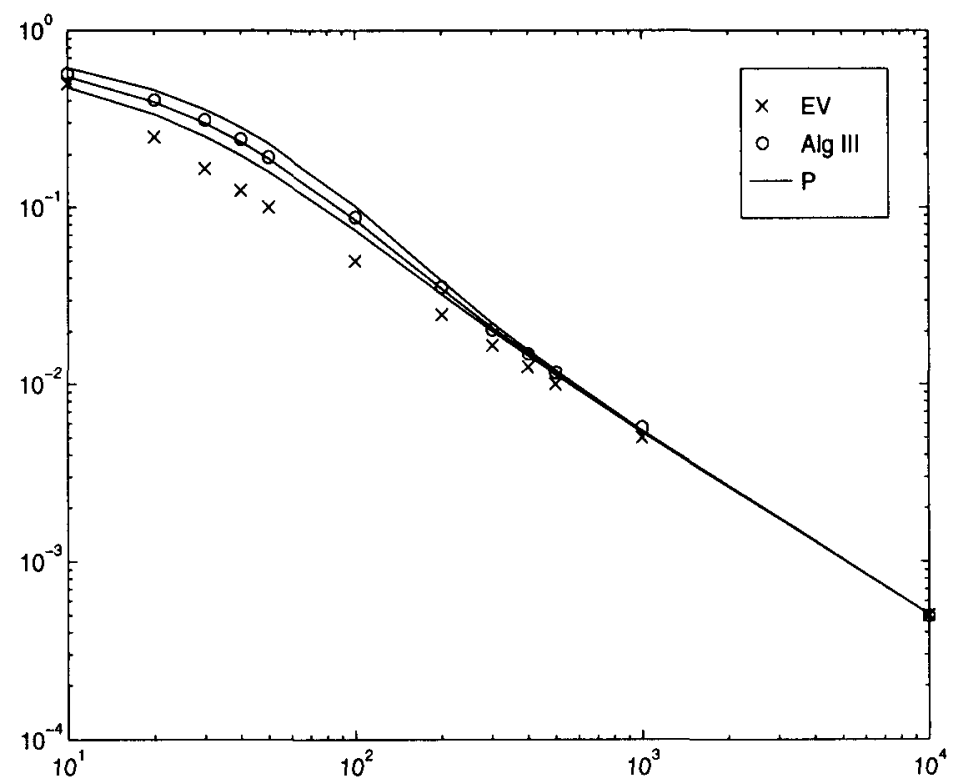

Figure 2: PAR(1,2), $\theta=0.1$

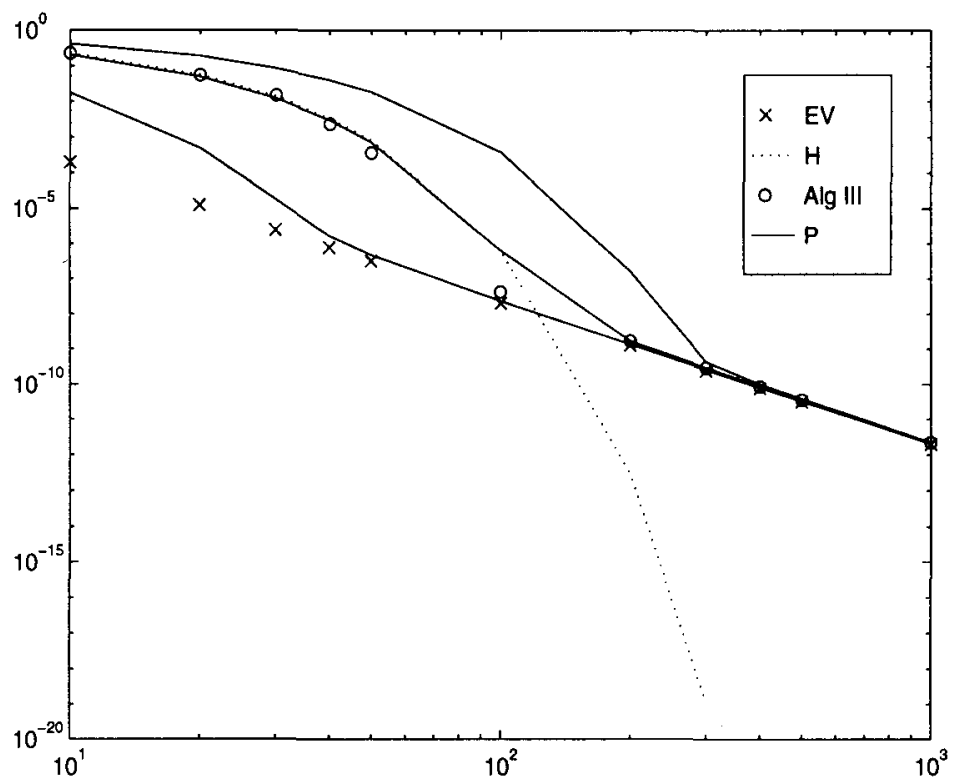

Figure 3: PAR(1,5), $\theta=0.1$ 


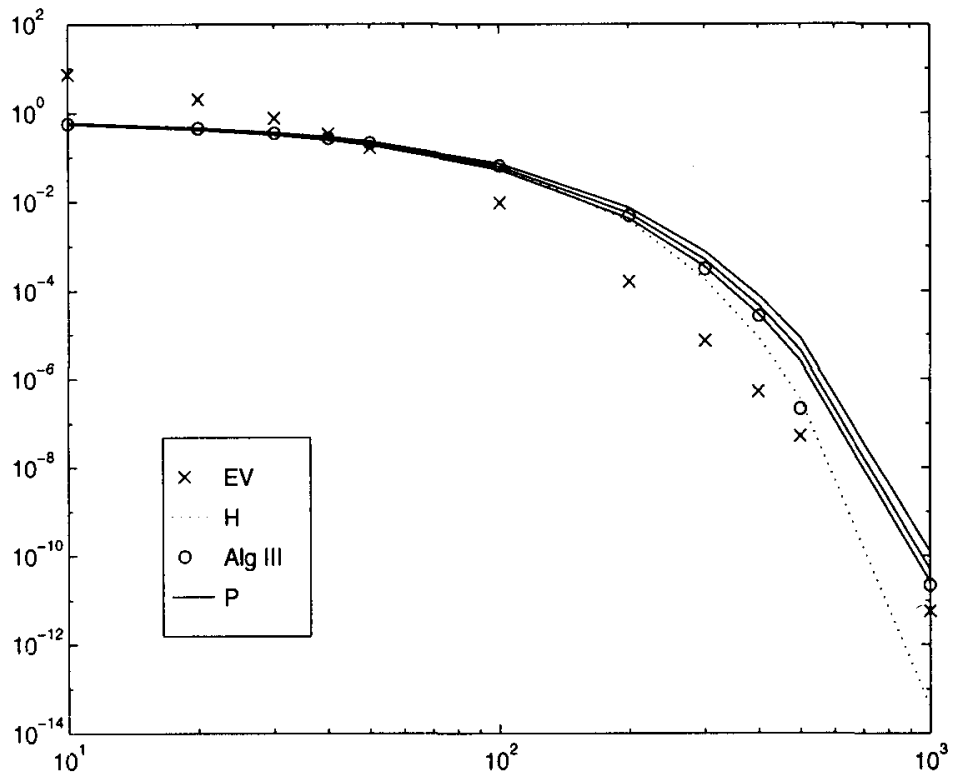

Figure 4: Weibull(1/2), $\theta=0.2$

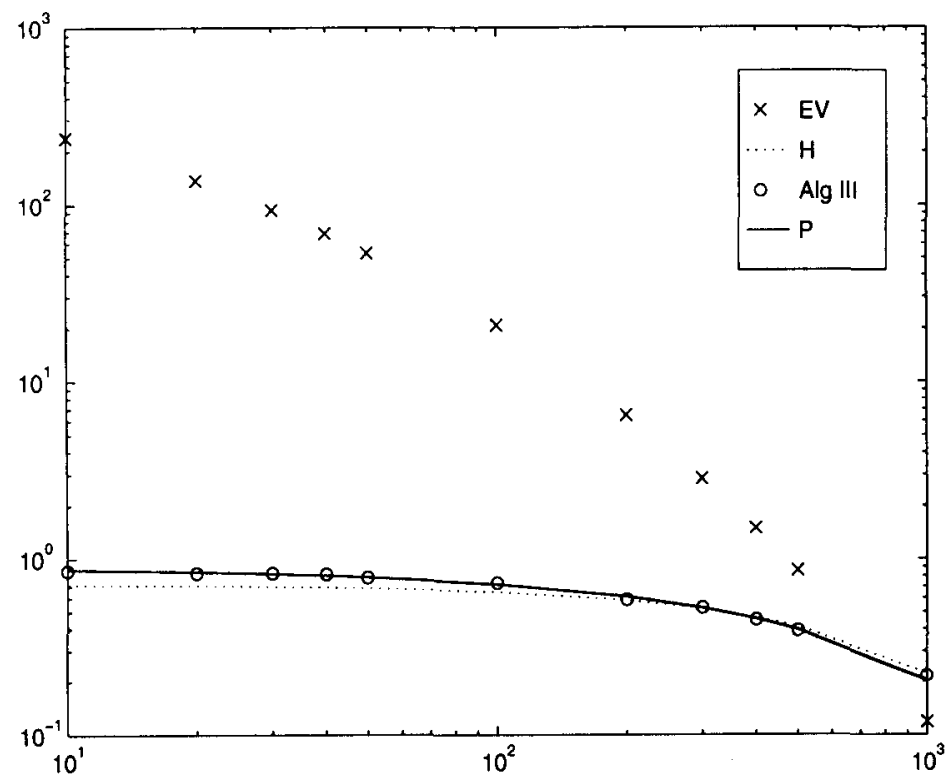

FIGURE 5: Weibull(1/3), $\theta=0.1$ 
Thorin and Wikstad [31] have calculated the exact ruin probabilities for some values of $u$ and $\theta$. Therefore we compare our estimates with those values.

4.5. Results. The Tables 1-3 contain the estimates for different initial reserve $u$ derived from the three algorithms together with their confidence intervals and the precision measured by $(2)$. The estimates for PAR $(1,2)$ distributed claims with security loading $\theta=0.1$ are presented in Table 1 . The results for PME(3) distributed claims with $\theta=0.25$ are shown in Table 2 and for Lognormal $(-1.62,1.8)$ claims with $\theta=0.1$ in Table 3 .

In the Figures 1-5 we give the simulated values from Algorithm III based upon $n$ $=200$ replications, the approximation $\psi_{E V}(u)$ and $\psi_{H}(u)$ (if the third moment exists). These values are compared with the estimates, lower and upper bound derived from Panjer's approximation. Figure 1 shows the values for $\operatorname{PAR}(1,1.5)$, $\theta=0.3$, Figure 2 for $\operatorname{PAR}(1,2), \theta=0.1$ and Figure 3 for $\operatorname{PAR}(1,5), \theta=0.1$. For the Weibull distribution we give the figure for $v=1 / 2, \theta=0.2$ and for $v=1 / 3, \theta=0.1$.

\section{CONCLUSION}

Below we give an overview of the most important properties of the algorithms and approximations we considered. The key observations from the above tables and figures as well as other examples, see Binswanger [8], are:

O1 Algorithm I works fine for 'small' initial capital and underestimates $\psi(u)$ when $u$ is 'large'.

O2 Algorithm II usually overestimates $\psi(u)$ for 'small' $u$ and underestimates for 'large' $u$.

O3 Algorithm III is always of the right order of magnitude.

O4 The precision measured by (2) is usually around $\frac{1}{2}$ for Algorithm 1. For Algorithm II it is also around $\frac{1}{2}$ as long as the estimates are valid and around 1 when the estimates are wrong. The precision of the third algorithm is always around 1 even when the claim size distribution is Weibull.

O5 The corrected diffusion approximation (13) gives very satisfactory results for 'small' initial capitals and is poor for 'large' initial reserves. The less heavy tailed the distribution of the claims is, the better the approximation is.

O6 The asymptotic approximation (6) often requires $u$ to be so large that the resulting ruin probability becomes extremely small, in fact much smaller than typical values of practical interest. The approximation turns out to be better the more heavy-tailed $B$ is. In particular, it is much better for Pareto then for Weibull distributed claims.

Of course it would be nice to know what 'large' and 'small' initial capitals mean. The interpretation of 'large' or 'small' depends on the kind of distribution and on the choice of its parameter as well as on the security loading $\theta$. 
A comparative study of the accuracy of the various bounds and approximations in De Vylder and Goovaerts [10], Dickson [12], Omey and Willekens [24] and Omey and Willekens [25] is given by Binswanger [8]. In the latter, also alternative variance reduction techniques, like the use of regression-adjusted control variates, are to be found.

We point out also that Algorithm III applies to the total claims as well. That is, rather than the ruin probability, one wants to compute

$$
G(x)=P\left(\sum_{i=1}^{M} \xi_{i}>x\right)
$$

by simulation where $M$ is the number of claims in a given period. The simplest case is where $M$ is Poisson with parameter $\lambda$, say, and one can proceed just as for the ruin probability, generating $M$ as Poisson rather than geometric. One again obtains the efficiency property (2). More generally, $M$ could be allowed to have any distribution with finite second moment. For example, one could treat risk processes where the arrivals occur according to some Cox process in this way.

Besides Panjer's recursion also transform inversion via FFT offers an interesting estimation method. See for instance Embrechts, Grübel and Pitts [14] and Buchwald, Chevallier and Klüppelberg [9] for a discussion in the context of insurance.

For a broad overview of the application of numerical methods in risk theory, see Feilmeier and Bertram [17].

\section{ACKNOWLEDGEMENT:}

The authors would like to thank a referee for various remarks on earlier versions of the paper.

\section{REFERENCES}

[1] Aвate, J., Choudhury, G. L., and Whitt, W. Waiting-time tail probabilities in queues with longtailed service-time distributions. Queueing Systems 16 (1994), 311-338.

[2] ABate, J., and WHITT, W. The Fourier-series method for inverting transforms of probability distributions. Queueing Systems 10 (1992), 5-88.

[3] Arnold, B. C., Balakrishnan, N., and Nagaraja, H. N. A First Course in Order Statistics. Wiley, New York, 1992.

[4] ASMUSSEN, S. Conjugate processes and the simulation of ruin problems. Stochostic Processes and their Applications 20 (1985), 213-229.

[5] Asmussen, S. Applied Probability and Queues. Wiley, Chichester, 1987.

[6] Asmussen, S. Approximations for the probability of ruin within finite time. Scandinavian Actuarial Journalp 31-57, 1984, (p:64,1985).

[7] Asmussen, S., and Rubinstein, R. Steady-state Rare Events Simulation in Queueing Models and its Complexity Properties, Advances in Queueing (J. Dhashalou, ed), 79-102. CRC Press, Boca Raton, Florida, 1995.

[8] Binswanger, K. Rare Events and Insurance. ETH Zürich, PhD-thesis No. 12233.

[9] Buchwald, M., Chevallier, E., and Klüppelberg, C. Approximation methods for the total claimsize distribution - an algorithmic and graphical presentation. Mitteilungen der Schweizerischen Vereinigung der Versicherungsmathematiker 2 (1993), 187-226. 
[10] De Vylder, F., and Goovaerts, M. Bounds for classical ruin probabilities. Insurance: Mathematics and Economics 3 (1984), 121 - 131.

[11] Devroye, L. Non-Uniform Random Variate Generation. Springer-Verlag, New York, 1986.

[12] Dickson, D. C. M. An upper bound for the probability of ultimate ruin. Scandinavian Actuarial Journal 2 (1994), 131-138.

[13] Dickson, D. C. M. A review of Panjer's recursion formula and its applications. British Actuarial Journall (1995), 107-124.

[14] Embrechts, P., Grübel, R., and Pitts, S. Some applications of the fast Fourier transform algorithm in insurance mathematics. Statistica Neerlandica 47 (1993), 59-75.

[15] Embrechts, P., and KlüpPElberg, C. Some aspects of insurance mathematics. Theory of Probability and its Applications 39 (1994), 262-295.

[16] Embrechts, P., and Veraverbeke, N. Estimates for the probability of ruin with special emphasis on the possibility of large claims. Insurance: Mathematics and Economics I (1982), 55-72.

[17] Feilmeier, M., and Bertram, J. Anwendungen numerischer Methoden in der Risikotheorie. Verlag Versicherungswirtschaft E. V., Karlsruhe, 1987.

[18] GlynN, P. W., and IGLehaRT, D. L. Importance sampling for stochastic simulations. Management Science 35 (1989), 1367-1392.

[19] GrandeLl, J. A class of approximations of ruin probabilities, Scandinavian Actuarial Journal Suppl. (1977; 1978: 77-78, 1978), 1977: 37-52.

[20] Heidelberger, P. Fast simulation of rare events in queueing and reliability models. ACM Trans actions on Computer Modeling and Simulation 5 (1995), 43-85.

[21] HogAN, M. L. Comment on corrected diffusion approximations in certain random walk problems. Journal of Applied Probability 23 (1986), 89-96.

[22] IGLEHART, D. L. Diffusion approximations in collective risk theory. Journalof Applied Probability $6(1969), 285-292$.

[23] KLÜPPELBERG, C. Estimation of ruin probabilities by means of hazard rates. Insurance: Mathematics and Economics 8 (1989), 279-285.

[24] OMEY, E., and WILLEKENS, E. Second order behaviour of the tail of a subordinated probability distribution. Stochastic Processes and their Applications 21 (1986), 339-353.

[25] OMEY, E., and WILlEKENS, E. Second order behaviour of distributions subordinate to a distribution with finite mean. Communications in Statistics; Stochastic Models 3(3) (1987), 311 342.

[26] Panjer, H., and Doray, L. Further results on the probability of ruin with an absorbing upper barrier. Transactions of the 23rd International Congress of Actuaries Helsinki (1988), 159-177.

[27] Panjer, H. H., and Willmot, G. E. Insurance Risk Models. Society of Actuaries, 1992.

[28] Rubinstein, R. Y. Simulation and the Monte Carlo Method. Wiley, New York, 1981.

[29] Sibgmund, D. Importance sampling in the Monte Carlo study of sequential tests. The Annals of Statistics 4 (1976), 673-684.

[30] Siegmund, D. Corrected diffusion approximations in certain random walk problems. Advances in Applied Probability 11 (1979), 701-719.

[31] THORIN, O., and WIKSTAD, N. Calculation of the ruin probabilities when the claim distribution is lognormal. ASTIN Bulletin 9 (1979), 231-246.

\section{S. ASMUSSEN}

Department of Mathematical Statistics

University of Lund

S-22100 Lund

Sweden

K. Binswancer

Departement of Mathematics

ETH Zurich

CH-8092 Zurich

Switzerland 